\section{Effect of shock intensity on the retention of an avoidance response*}

\author{
JOHN BINTZ \\ University of California, Davis, Calif. 95616
}

Three groups of 60 rats were given 40 trials of standard shuttle-avoidance conditioning with a tone $\mathrm{CS}$ and a shock intensity of $.4, .8$, or $1.6 \mathrm{~mA}$. Within each group, four subgroups were matched on the number of avoidances during acquisition and given retention tests of 20 trials at $5 \mathrm{~min}, 1,4$, or $24 \mathrm{~h}$ after training. The terminal level of acquisition performance was an inverted-U-shaped function of the shock intensity, and performance during retention tests was a U-shaped function of the retention interval, with the minimum occurring at $1 \mathrm{~h}$ for the $.8-\mathrm{mA}$ shock group and at $4 \mathrm{~h}$ for the .4 - and 1.6-mA shock groups.

In many situations and for a variety of species, the retention function of an aversively motivated response is a U-shaped function of the retention interval (Kamin, 1957; Pinckney, 1966; Bintz, Braud, \& Brown, 1970). The processes which mediate this function are not well understood except that the nonmonotonicity appears to reflect the association of the CS with the UCS, or fear, rather than any instrumental contingency (Brush, Myer, \& Palmer, 1963). Apparently, the amount of fear elicited by the CS declines directly after training and subsequently increases (Bintz et al, 1970). Additionally, it appears that the deficiency at intermediate retention intervals represents a retrieval rather than a motivational deficit (Bintz, 1970; Klein \& Spear, 1970).

One of the persistent sources of variance in studies of this retention function is the point in time at which the minimum is found and has been shown at intervals ranging from 1 to $24 \mathrm{~h}$ after training. It has been hypothesized (Brush, Myer, \& Palmer, 1964; Brush, 1970) that the minimum of the function is inversely related to the degree of conditioning. When a criterial design is employed for original training and the number of trials required to reach the criterion is manipulated either by experimental parameter (e.g., intertrial interval; Brush et al, 1964) or by dividing at the median between fast and slow learners (Kamin, 1963), then the group that requires the most trials reaches a minimum later than does the group that requires fewer trials. When a constant-trials

*This report is based on a portion of a dissertation submitted to the Graduate School of the University of Iowa in partial fulfillment of the requirements for the $\mathrm{PhD}$ degree. The author is indebted to Judson S. Brown for his advice and consultation throughout the course of the investigation, as well as to I. Gormezano, D. J. Levis, R.W. Schulz, and D.W. McAdam. Requests for reprints should be sent to John Bintz, Department of Psychology, University of California, Davis, California 95616. design is employed for original training (all conditions of training the same, except that different groups receive a different number of trials), the group given the most trials reaches a minimum earlier than does the group given fewer trials (Brush, 1963).

Thus, the retention interval at which the minimum occurs is both directly and inversely related to the number of trials, depending upon the type of design employed; but in all cases, the relation between the retention-interval minimum and the degree of conditioning is invariant and inverse. Since fear conditioning is both necessary and sufficient and the instrumental contingencies make little difference in producing the basic effect (Brush et al, 1963), it has been further argued that in each of the above cases the relevant variable is the degree of original fear conditioning (Brush, 1970) rather than the degree of instrumental conditioning. This explanation runs into some difficulty in accounting for the Kamin (1963) and the Brush et al (1964) results, since it must be assumed that those Ss which learn to avoid in the fewest trials must have the greatest degree of conditioned fear, despite the fact that they have received fewer fear-conditioning trials in the course of learning to avoid. In the present experiment, avoidance retention is studied as a function of shock intensity, a variable known to produce large differences in degree of both fear and avoidance conditioning.

\section{SUBJECTS}

The Ss were 180 experimentally naive male hooded rats of the Long-Evans strain purchased from the Blue Spruce Farms at Altamont, New York. They weighed 200-250 g and were an estimated 70-100 experiment. Throughout the course of the experiment, they were housed in individual cages and maintained on an ad lib feeding schedule.

\section{APPARATUS}

The major piece of equipment was a days old at the beginning of the rectangular hurdlebox which was divided into two identical compartments by a metal barrier 2 in. high. Each of the compartments was 11 in. long, $5 \frac{1}{2} \mathrm{in}$. wide, and $10 \mathrm{in}$. high inside. Three sides were constructed of varnished wood, while the top and front were of Plexiglas. The floor of the apparatus consisted of stainless steel bars, $3 / 32$ in. in diam, spaced at 1/2-in. intervals.

The UCS was a constant-current electric shock of $.4, .8$, or $1.6 \mathrm{~mA} \mathrm{dc}$ (measured with 150,000 ohms in the circuit), which was scrambled by a Lehigh Valley scrambling device. The CS was a steady tone of $2,000 \mathrm{~Hz}$, delivered through two identical speakers located on each end wall. Crossing responses were indicated by the depression of microswitches, located under the grid, by the weight of $S$. The hurdlebox was situated in a refrigerator shell with the associated programming equipment outside. A $71 / 2-\mathrm{W}$ light was located in the refrigerator as a houselight.

\section{PROCEDURE}

Three groups of $60 \mathrm{Ss}$ were given 40 trials of standard avoidance training with a UCS intensity of $.4,8$, or $1.6 \mathrm{~mA}$. Trials were given with an interval of $45 \mathrm{sec}$ between the response terminating one trial and the onset of the CS commencing the next trial. If $\mathrm{S}$ made no response, the trial was automatically terminated $30 \mathrm{sec}$ after CS onset. The interval separating the CS and UCS was $5 \mathrm{sec}$, and both were response terminated when $\mathrm{S}$ crossed the barrier.

Within each intensity level, four subgroups of $15 \mathrm{Ss}$ each were matched on the number of acquisition avoidances. These four subgroups, at each intensity, were subsequently given 20 avoidance trials at retention intervals of $5 \mathrm{~min}, 1,4$, or $24 \mathrm{~h}$ after training. All conditions of testing were identical with the conditions of training, including shock intensity for each S. During the retention interval, each $S$ was placed in its home cage for the appropriate amount of time.

\section{RESULTS}

Acquisition

The percentage of avoidance responses as a function of trials is plotted for each of the three shock intensities in Fig. 1. During the early trials, performance was an inverse function of shock intensity, but on later trials the .8-mA group surpassed the .4-mA shock group. The strong-shock group did not start learning as quickly as did either the weak- or medium-shock groups, but appeared to learn about as rapidly once it began. Analyses of variance indicate that the difference between shock-intensity groups was highly significant $[F(92,177)=16.20, p<.001]$, as was the interaction between trial blocks and shock intensity $[F(14,1239)=3.99, p<.001]$. In specific contrast comparisons, it was 


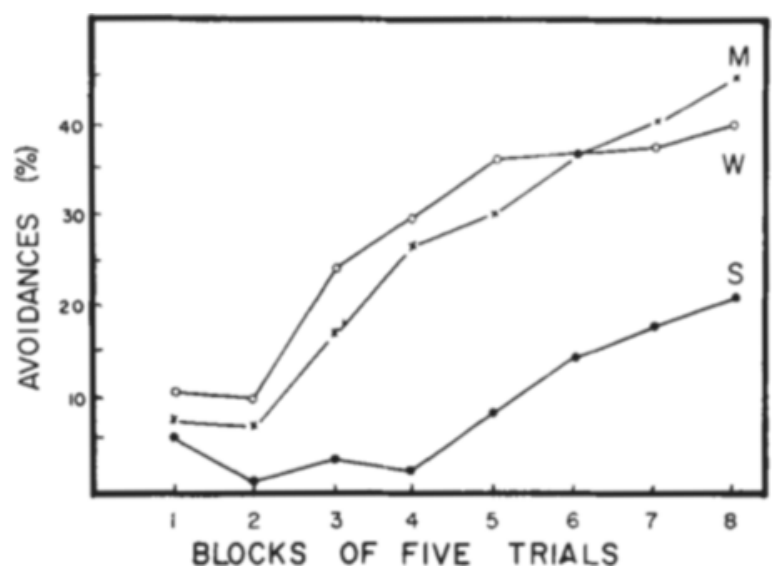

Fig. 1. Percentage of avoidance responses as a function of trials for strong-, medium-, and weak-UCS-intensity groups.

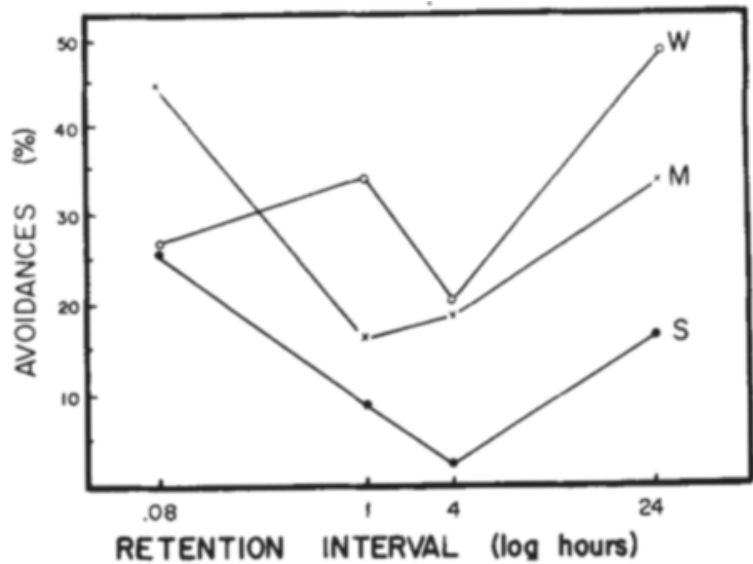

Fig. 2. Percentage of avoidance responses as a function of the retention interval for strong-, medium-, and weak-UCS-intensity groups. found that neither the shock-intensity effect nor the interaction between intensity and trials was significant for the comparison between weak and medium groups. However, when the strong-shock group was compared with either the weakor medium-shock group, both the main effects $(p<.001)$ and the interactions $(\mathrm{p}<.001)$ were significant.

\section{Retention}

Mean percentages of avoidance responses as a function of the retention interval are plotted for each level of UCS intensity in Fig. 2. Except for the 5-min group in the weak-shock condition, regular-appearing Kamin-type functions were found at each level of UCS intensity. The minimum appeared at $4 \mathrm{~h}$ for both the weak- and strong-shock groups and at $1 \mathrm{~h}$ for the medium-shock group. The main effects of both the retention interval $[F(3,168)=34.29, \quad p<.005]$ and the shock intensity $[F(2,168)=57.40$, $\mathrm{p}<.001]$ were significant.

\section{DISCUSSION}

The acquisition results of this experiment are consistent with findings of other studies in which shock intensity has been found to be inversely related to avoidance proficiency (Moyer \& Korn, 1964; Levine, 1966). That there is no difference between weak and medium shock-intensity groups in acquisition is surprising. It might be that the function is actually flat in this range, but this is unlikely, since other studies have found differences throughout the shock-intensity dimension. It might be a sampling error, but this too appears unlikely with a sample size of 60 Ss per group. It is more probable that the values of .4 and $.8 \mathrm{~mA}$ fell on functions moving in opposite directions, and some intermediate value, such as $.6 \mathrm{~mA}$, would have resulted in better avoidance than either of the values used in this study. Whereas most studies have demonstrated an inverse effect of shock intensity throughout the entire dimension tested, it must be the case that a small portion of the function is direct, since at the extreme case of $0 \mathrm{~V}$, avoidance over and above the appropriate controls should not occur.

It appears from these data that the best UCS intensity at which to study the Kamin effect is in the intermediate range. An intensity that is too high results in poor avoidance, and a great deal of power is lost because many Ss contribute nothing to the data pool, i.e., have zero avoidances. If the intensity is too low, irregularities appear in the data, which may or may not be attributable to the same processes that underlie the Kamin effect. While there is a Kamin-type minimum at the 4- $h$ interval in the weak-shock group, there is also a decrement in performance at the 5 -min interval. The same type of effect has been found previously with weak-shock groups (Bintz, 1966; Brush ${ }^{1}$ ), although it has not previously been reported. The 5-min group most nearly represents continued training (and, in fact, similar functions were found with continued training by Bintz, 1966), and the decrement may, therefore, be a maintenance problem associated with weak shock (D'Amato, Fazzaro, \& Etkin, 1967). These data tend to support an instrumental version of the degree-of-conditioning hypothesis. The group with the highest terminal level of performance at the end of acquisition trials (the medium-shock group) reaches a minimum at $1 \mathrm{~h}$ during retention tests, whereas both the weak- and the strong-shock groups reach a minimum at $4 \mathrm{~h}$. However, this conclusion must be somewhat tenuous, since there is little difference between the weak- and medium-shock groups at the end of acquisition and there is little difference in the performance of the medium-shock group at 1 - and 4 -h retention intervals.

\section{REFERENCES}

BINTZ, J. J. The effect of response strength on the retention of an aversively motivated response. Unpublished MA thesis, University of Iowa, 1966.

BINTZ, J. Time-dependent memory deficits of aversively motivated behavior. Learning \& Motivation, 1970

BINTZ, J., BRAUD, W. G., \& BROWN, J. S. An aversively motivated behavior. Learning \& Behavior, in press.

BRUSH, F. R. Some variables influencing transfer from classical fear conditioning to instrumental avoidance training. Paper presented at the meeting of the Psychonomic Society, Bryn Mawr, Pennsylvania, 1963.

BRUSH, F. R. Retention of aversively motivated behavior. In F. R. Brush (Ed.), Aversive conditioning and learning. New York: Academic Press, 1970.

BRUSH, F. R., MYER, J. S., \& PALMER, M. E. Effects of kind of prior training and intersession interval upon subsequent avoidance learning. Journal of Comparative \& Physiological Psychology, 1963, 56.539-545.

BRUSH, F. R., MYER, J. S., \& PALMER, M. E. Joint effects of intertrial and intersession interval upon avoidance learning. Psychological Reports, 1964, 14, 31-37.

D'AMATO, M. R., FAZZARO, J., \& ETKIN, M. Discriminated bar-press avoidance maintenance and extinction in rats as a function of shock intensity. Journal of Comparative \& Physiological Psychology, 1967, 63, 351-354.

KAMIN, L. J. Retention of an incompletely learned avoidance response. Journal of Comparative \& Physiological Psychology, 1957, 50, 457-460.

KAMIN, L. J. Retention of an incompletely learned avoidance response: Some further analyses. Journal of Comparative \& Physiological Psychology, 1963, 56, 713-718.

KLEIN, S. B., \& SPEAR, N. E. Forgetting by the rat after intermediate intervals ("Kamin effect") as a retrieval failure. Journal of Comparative \& Physiological Psychology, 1970, 71, 165-170.

LEVINE, S. UCS intensity and avoidance learning. Journal of Experimental Psychology, 1966, 71, 163-164.

MOYER, K. E., \& KORN, J. H. Effect of UCS intensity on the acquisition and extinction of avoidance response. Journal of Experimental Psychology, 1964, 67, 352-359.

PINCKNEY, G. A. The Kamin effect in fish. Psychonomic Science, 1966, 4, 387-388.

1. Brush, F. R. Personal communication, 1970. 